\title{
Observation of an Excitonic Mott Transition through Ultrafast Core-cum-Conduction Photoemission Spectroscopy
}

\author{
Maciej Dendzik $\odot,{ }^{1,2, \dagger}$ R. Patrick Xian $\odot,{ }^{1}$ Enrico Perfetto, ${ }^{3,4}$ Davide Sangalli $\odot,{ }^{3,5}$ Dmytro Kutnyakhov, ${ }^{6}$ Shuo Dong $\odot,{ }^{1}$ \\ Samuel Beaulieu $\odot,{ }^{1}$ Tommaso Pincelli, ${ }^{1}$ Federico Pressacco $\odot,{ }^{7}$ Davide Curcio, ${ }^{8}$ Steinn Ymir Agustsson $\odot,{ }^{9}$ \\ Michael Heber, ${ }^{6}$ Jasper Hauer, ${ }^{1}$ Wilfried Wurth, ${ }^{6,7}{ }^{*}$ Günter Brenner, ${ }^{6}$ Yves Acremann, ${ }^{10}$ Philip Hofmann, ${ }^{8}$ Martin Wolf, ${ }^{1}$ \\ Andrea Marini $\odot{ }^{3}$ Gianluca Stefanucci®, ${ }^{4,11}$ Laurenz Rettig $\odot,{ }^{1}$ and Ralph Ernstorfer $\oplus^{1, \#}$ \\ ${ }^{1}$ Fritz Haber Institute of the Max Planck Society, Faradayweg 4-6, 14915 Berlin, Germany \\ ${ }^{2}$ Department of Applied Physics, KTH Royal Institute of Technology, Hannes Alfvéns väg 12, 114 19 Stockholm, Sweden \\ ${ }^{3}$ CNR-ISM, Division of Ultrafast Processes in Materials (FLASHit), Area della Ricerca di Roma 1, \\ Via Salaria Km 29.3, I-00016 Monterotondo Scalo, Italy \\ ${ }^{4}$ Department of Physics, Tor Vergata University of Rome, Via della Ricerca Scientifica 1, 00133 Rome, Italy \\ ${ }^{5}$ Department of Physics, University of Milan, via Celoria 16, I-20133 Milan, Italy \\ ${ }^{6}$ DESY Photon Science, Notkestr. 85, 22607 Hamburg, Germany \\ ${ }^{7}$ Center for Free-Electron Laser Science CFEL, Hamburg University, Luruper Chausee 149, 22761 Hamburg, Germany \\ ${ }^{8}$ Department of Physics and Astronomy, Interdisciplinary Nanoscience Center (iNANO), Aarhus University, \\ Ny Munkegade 120, 8000 Aarhus C, Denmark \\ ${ }^{9}$ Institute of Physics, Johannes Gutenberg University Mainz, D-55128 Mainz, Germany \\ ${ }^{10}$ Department of Physics, Laboratory for Solid State Physics, ETH Zurich, Otto-Stern-Weg 1, 8093 Zurich, Switzerland \\ ${ }^{11}$ INFN, Sezione di Roma Tor Vergata, Via della Ricerca Scientifica 1, 00133 Rome, Italy
}

(Received 19 March 2020; revised 7 June 2020; accepted 24 July 2020; published 24 August 2020)

\begin{abstract}
Time-resolved soft-x-ray photoemission spectroscopy is used to simultaneously measure the ultrafast dynamics of core-level spectral functions and excited states upon excitation of excitons in $\mathrm{WSe}_{2}$. We present a many-body approximation for the Green's function, which excellently describes the transient core-hole spectral function. The relative dynamics of excited-state signal and core levels clearly show a delayed core-hole renormalization due to screening by excited quasifree carriers resulting from an excitonic Mott transition. These findings establish time-resolved core-level photoelectron spectroscopy as a sensitive probe of subtle electronic many-body interactions and ultrafast electronic phase transitions.
\end{abstract}

DOI: 10.1103/PhysRevLett.125.096401

Optoelectronic properties of semiconductors are largely governed by two types of excitations-excitons [1], the bosonic quasiparticles comprised of an electron and a hole bound by Coulomb interaction, and quasifree carriers (QFCs) of single-particle character [2,3]. While the interplay between excitons and QFCs has been studied experimentally with terahertz and optical spectroscopies [4,5], these techniques are restricted to optically allowed transitions and do not provide direct information about the underlying many-body interactions. In this Letter, we show that detailed information about the dynamics of both excitons and QFCs can be deduced from the simultaneous measurement of the core-hole spectral function and the excited state population with ultrafast time-resolved $\mathrm{x}$-ray photoelectron spectroscopy (trXPS) [6]. We observe strong

Published by the American Physical Society under the terms of the Creative Commons Attribution 4.0 International license. Further distribution of this work must maintain attribution to the author(s) and the published article's title, journal citation, and DOI. Funded by Bibsam. renormalization of the $\mathrm{W} 4 f$ spectral function after optical excitation of $\mathrm{WSe}_{2}$ bulk crystals. The transient spectral function is excellently reproduced using a many-body approximation for the Green's function [7], which accounts for the core-hole screening by photoexcited QFCs. The simultaneous measurement of the excited-state population in the conduction band $(\mathrm{CB})$ reveals a $\sim 100$ fs delay of the core-hole screening compared to the initial buildup of the exciton population, which we ascribe to an ultrafast Mott transition from optically prepared excitons to an uncorrelated QFC plasma.

Static XPS has been a workhorse of surface science by driving the understanding of catalytic processes [8-10], chemical states of interfaces [11], and functional materials [12]. The measured photoelectron distribution is proportional to the core-hole spectral function and carries information about the many-body interactions such as Auger scattering, electron-phonon coupling, plasmonic excitations, and local screening [13-16]. The XPS line shape of metals is usually asymmetric and phenomenologically well described by the Doniach-Šunjić (DS) function [17], where the characteristic heavy tail towards 
higher binding energy originates from the core-hole screening by conduction electrons. For semiconductors, on the other hand, the observed line shape is typically symmetric and can be described by a Voigt profile $[18,19]$. In the presence of excited carriers, a semiconductor becomes partially metallic and one can expect a renormalization of the core-hole line shape. This opens up the possibility of studying nonequilibrium dynamics with XPS. Recently, technological advances of femtosecond X-ray sources [20] and photoelectron detectors [21] have made ultrafast trXPS experiments possible. These include the observation of melting of charge-density wave states in Mott insulators $[22,23]$, charge-transfer dynamics at semiconductor interfaces [24] or transient surface-photovoltage control [25]. In this work, we show a potential for trXPS studies of excitonic Mott transition, which is important for understanding the fundamental processes behind the dynamics of optically excited semiconductors. This is especially relevant to 2D materials, such as transition metal dichalcogenides (TMDCs), in which excitonic effects dominate low-energy behavior due to large exciton binding energy and reduced dielectric screening. However, an accurate theoretical description of the out-of-equilibrium core-hole spectral function is still missing and direct application of the DS theory to the dynamic case is problematic. In this work, we generalize the DS theory to cover the case of a photoexcited semiconductor, which enables a quantitative description of the fundamental processes governing the experimentally observed core-hole spectral changes.

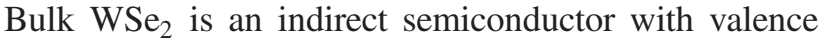
and conduction edges situated at $\bar{\Gamma}$ and $\bar{\Sigma}$, respectively [26]. The direct band gap at $\bar{K}$ points enables an efficient optical excitation of excitons and QFCs. Previous time- and angleresolved photoemission spectroscopy experiments revealed ca. $90 \mathrm{meV}$ energy difference between these species and scattering of the excited states population towards global conduction band minimum at $\bar{\Sigma}$ on the timescale of ca. $15 \mathrm{fs}$ [27]. We performed core-cum-conduction trXPS experiments of $\mathrm{WSe}_{2}$ using the FLASH free-electron laser and optical pump pulses tuned to the optical $A$-exciton resonance at $1.6 \mathrm{eV}$ at room temperature. A time-of-flight (TOF) momentum microscope was used as the photoelectron analyzer, which enabled us to simultaneously probe a $\sim 40 \mathrm{eV}$-broad spectral window including the excited states, valence band, and the highest $\mathrm{W}$ and $\mathrm{Se}$ core levels $[28,35]$. Schematics of the setup and a model conduction-core energy level diagram are shown in the Figs. 1(a)-1(b). The observed time-dependent trXPS spectra of $\mathrm{W} 4 f_{5 / 2}$ shown in Fig. 1(c) exhibit distinct dynamics with respect to pump-probe delay time, showing characteristic changes of photoemission peak position and width. In addition, we observed a buildup of asymmetry (skewness) that resembles the DS asymmetry. Simultaneously, we observed a transient population of excited carriers responsible for the core-hole line shape modifications.

In order to understand the origin of the observed trXPS spectral changes, we propose a theoretical model to

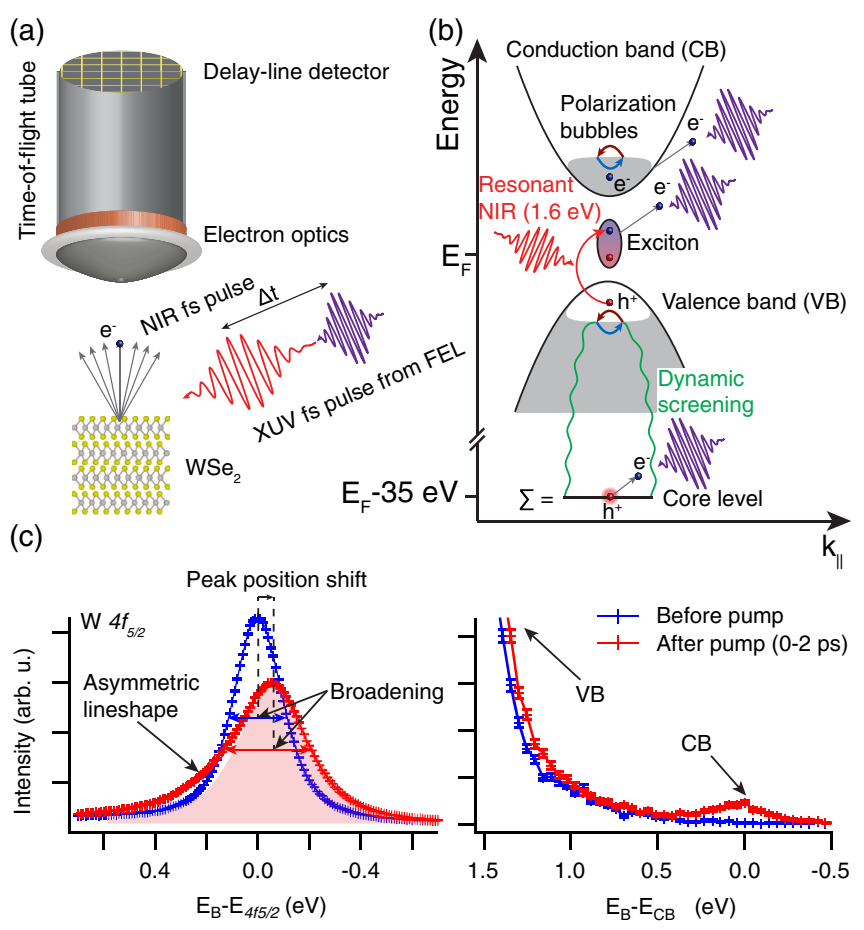

FIG. 1. (a) Sketch of the experimental setup for pump-probe trXPS on $\mathrm{WSe}_{2}$ using a momentum microscope, which enables the simultaneous analysis of core, valence, and conduction electrons. A sample is excited with a near-infrared (NIR) pulse and probed with an extreme ultraviolet (XUV) pulse. (b) Energy band diagram of the photoexcited $\mathrm{WSe}_{2}$ near the $\bar{K}$ valley: the NIR pulse (red) resonant with the exciton energy generates excited carrier populations in the valence $\left(h^{+}\right)$and conduction $\left(e^{-}\right)$bands. Transient conduction band population as well as the induced renormalization of the screened core-hole spectral function are detected by the XUV pulse (violet). The core linewidth is affected by the screening (polarization bubbles) generated by particle-hole excitations both in the valence and conduction bands, here included through the self-energy $\Sigma$. (c) Core-cum-conduction trXPS spectra showing W $4 f_{5 / 2}$ (left) and momentum-integrated conduction band (right) regions, before (blue) and after the excitation (red). Colored arrows indicate corresponding full width at half maximum of the spectra and the shaded area is the symmetric part of the line shape, illustrating its asymmetry.

describe the dynamical screening of the core hole due to the photoinduced valence holes and conduction electrons. We refer the reader to Ref. [28] for details. Briefly, the corelevel photoemission signal is proportional to the core-hole spectral function within the adiabatic approximation [14]

$$
A(\omega)=-\pi^{-1} \operatorname{Im}[G(\omega)],
$$

with $G(\omega)$ being the core-hole Green's function

$$
G(\omega)=\frac{1}{\omega-\epsilon_{c}-\Sigma(\omega)+i \gamma} .
$$

Here, $\epsilon_{c}$ is the core energy, $\Sigma(\omega)$ is the correlation selfenergy due to scatterings between the core electron and 

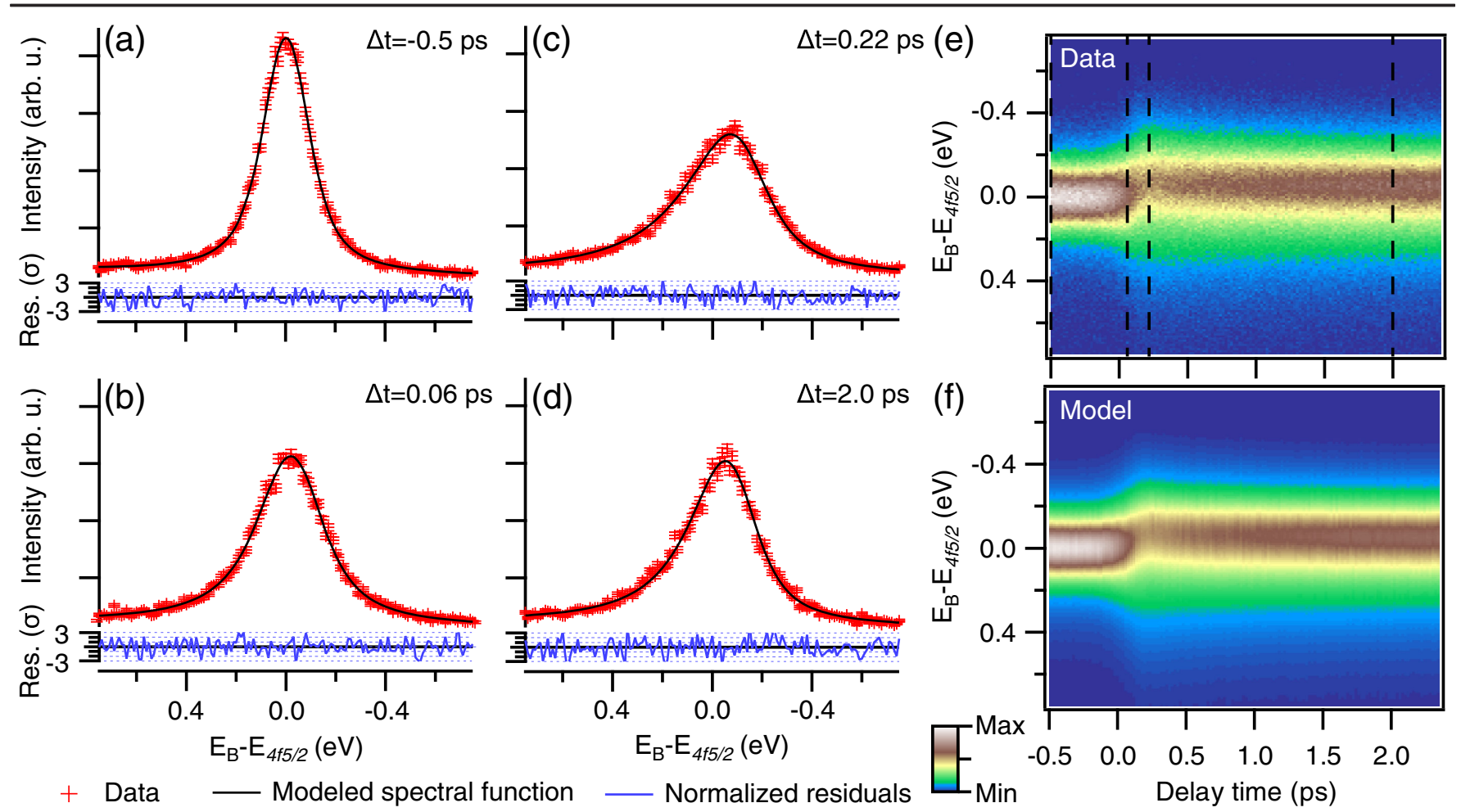

FIG. 2. (a) Representative energy spectra at four delay times marked in (e). Red points, black lines, and blue lines mark experimental data, fit result, and normalized residuals, respectively. Normalized residuals are presented in multiples of the data's Poisson distribution standard error. (e) trXPS spectrum of $\mathrm{W} 4 f_{5 / 2}$ as a function of pump-probe delay time. Photoemission intensity is encoded in the falsecolor scale. (f) Corresponding modeled spectral function, defined by Eqs. (1)-(4).

conduction or valence electrons, and $\gamma$ quantifies the broadening due to other decay channels, such as Auger or phonon scattering. According to the DS theory, the noninteracting line shape is mainly renormalized by dynamical screening effects [36,37]. In the diagrammatic formalism, this means that the self-energy $\Sigma(\omega)$ is dominated by the $G W$ term [38], where $W$ is the screened interaction in the random phase approximation. In this work, we show that screening the interaction with the single polarization bubble of QFCs [see Fig. 1(b)] is enough to reproduce the core-level shift and the asymmetric line shape. The screening due to excitons is much weaker in comparison to QFCs for TMDC materials [2] and it is therefore neglected. The resulting self-energy takes the form

$$
\Sigma(\omega)=\lambda \log \left(\frac{D}{\omega-\tilde{\epsilon}_{c}+i \gamma}\right)
$$

where $D$ is a parameter proportional to the average of conduction and valence bandwidths, while the renormalized core energy reads

$$
\tilde{\epsilon}_{c}=\epsilon_{c}+\lambda L\left(\frac{e^{\lambda / D} D}{\lambda}\right) ; \quad \lambda=\frac{m^{*}}{\pi} n_{\mathrm{QFC}} \nu^{2} .
$$

In Eq. (4), $L(x)$ is the Lambert function, $m^{*}$ is the effective mass at the band edge (the average value of conduction and valence band effective masses), $n_{\mathrm{QFC}}$ is the quasifree carrier density and $\nu$ is the average Coulomb interaction between the core electron and the valence or conduction electrons. In the absence of QFCs, $A(\omega)$ reduces to a Lorentzian profile with width dictated by $\gamma$, while at a finite QFC density, the real and imaginary parts of the self-energy are responsible for the shift of the core energy and the asymmetric line shape.

Qualitatively similar results of core-hole renormalization were observed for both $\mathrm{W} 4 f$ and Se $3 d$ states [35]. Although quantitative differences due to different screening interactions with excited carriers occur, the observed dynamics of these core-level states are consistent. Interestingly, Se $4 s$ states showed no renormalization effects [28], probably due to much shorter core-hole lifetime. Figure 2 presents the experimental data of $\mathrm{W} 4 f_{5 / 2}$ which can be excellently reproduced by the modeled spectral function [Eqs. (1)-(4)] convoluted with a constant Gaussian to account for the experimental energy resolution. This is evidenced by the featureless normalized residuals, shown for four representative time delays in Figs. 2(a)-2(d). The entire time series [see Figs. 2(e)-2(f)] can be reproduced by fixing the average bandwidth $D=0.8 \mathrm{eV}$ and effective mass $m^{*}=0.5$ (in units of the electron mass) of the material and solely fitting the broadening $\gamma(t)$ and the product $n_{\mathrm{QFC}}(t) \nu^{2}$ (the average interaction $\nu$ is independent of time) for every delay time $t$. 

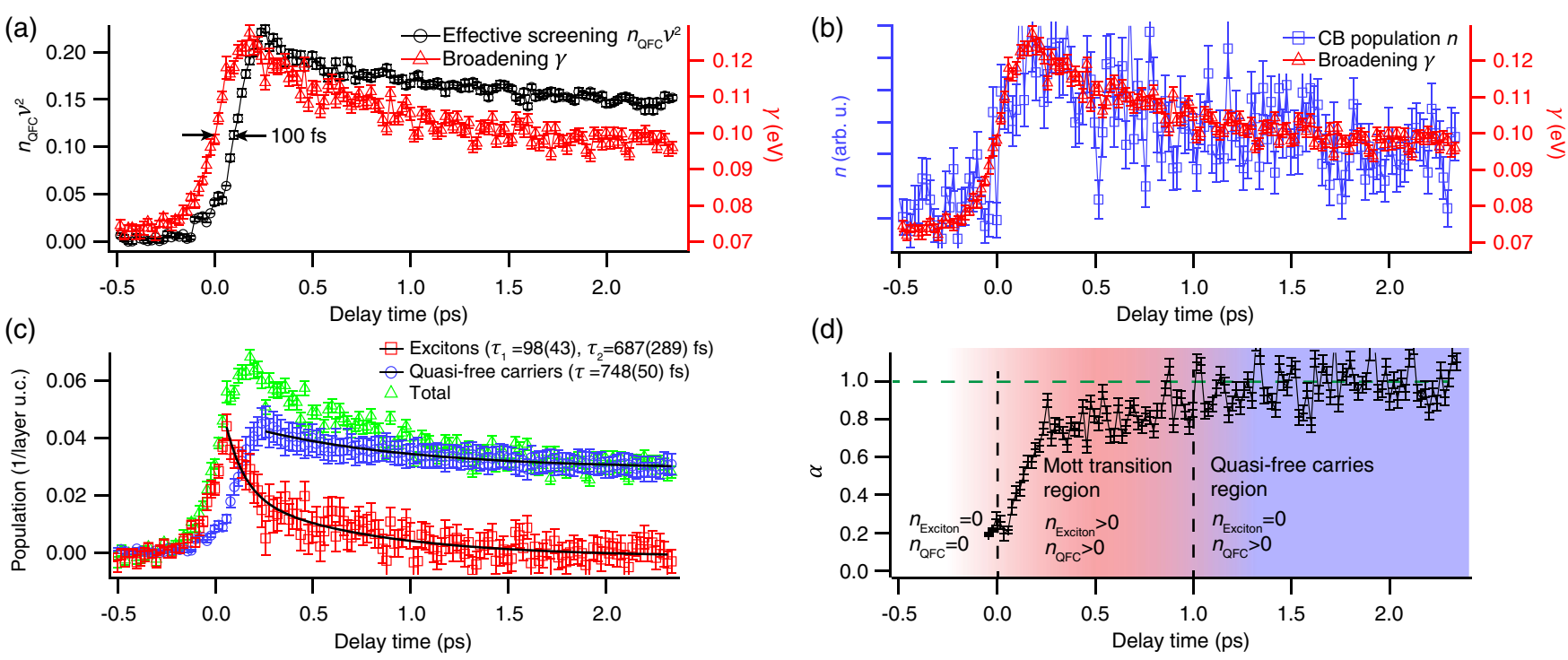

FIG. 3. (a) Comparison of the time-dependent effective screening $n_{\mathrm{OFC}} \nu^{2}$ (black circles) and broadening $\gamma$ (red triangles), obtained from fitting the theoretical model to the experimental data presented in Fig. 2. (b) Comparison of the photoemission signal above the Fermi level, corresponding to the $\mathrm{CB}$ population, (blue squares) and $\gamma$ (red triangles). (c) Decomposition of the dynamics of excitons (red squares), QFC (blue circles), and total excited carriers (green triangles). Black lines present fitted double exponential and single exponential decay for excitons and QFC, correspondingly. (d) The degree of ionization dynamics, as defined in the main text. Three different temporal regions are marked by the background color: before the excitation (white), Mott transition (red), and QFC regions (blue).

The transient spectral function can thus be described by just two independent parameters, as $n_{\mathrm{QFC}} \nu^{2}$ couples the experimentally observed peak shift and asymmetry of the line shape and $\gamma$ describes the symmetric broadening. Interestingly, these parameters exhibit drastically different dynamics, with $n_{\mathrm{QFC}} \nu^{2}$ rising ca. 100 fs later and decaying slower than $\gamma$ [see Fig. 3(a)]. This effect is not induced by the applied model, as the same behavior is present in modelindependent quantities such as the higher moments of the photoelectron distribution [28].

The TOF momentum microscope allows simultaneous detection of photoelectrons over a large energy range, spanning electrons from the core levels, valence band, and excited population in the $\mathrm{CB}$, within a single experiment (see Fig. 1). Therefore, it is possible to directly compare the dynamics of $\gamma$ with the buildup of the excited-state population $n$, and we find a strong correlation between the two quantities [see Fig. 3(b)], i.e., the core-level broadening immediately follows the buildup of excited carriers $n$ which includes contributions from both excitons [39] and QFCs in the CB. In contrast, the core-hole line shape renormalization governed by the quasiparticle screening $n_{\mathrm{QFC}} \nu^{2}$ shows a clear delay in buildup compared to $\gamma$ and $n$. This is consistent with the prediction that the pump energy tuned to the excitonic resonance should favor the creation of excitons [40] up to a critical density [41], and can be explained by means of an excitonic Mott transition - the initial stage of the dynamics is dominated by excitons which subsequently break into a QFC plasma due to increased screening as well as many-particle renormalizations [41]. An estimation of the excitation density per layer, $n=7(1.4) \times 10^{13} \mathrm{~cm}^{-2}$ [28], used in our experiment indeed significantly exceeds the predicted critical excitation density of approximately $3 \times 10^{12} \mathrm{~cm}^{-2}$ [2], and is close to the density of $1.1 \times 10^{14} \mathrm{~cm}^{-2}$ reported for experimental observation of excitonic Mott transition in single-layered $\mathrm{WS}_{2}$ [42].

The simultaneous acquisition of both excited states population in the whole surface Brillouin zone and renormalized core-hole spectral function excludes the effect of space charge, often observed in ultrafast photoemission experiments $[43,44]$, as space charge would not contribute to the $\mathrm{CB}$ population. We also exclude the influence of the interband $\bar{K}-\bar{\Sigma}$ scattering due to much faster dynamics of ca. 15 fs [27]. Moreover, we exclude surface-photovoltage observed before for $\mathrm{WSe}_{2}$ [25] as origin of the observed renormalization. This effect can influence the peak position, but not the asymmetry of the XPS spectra. In addition, the lack of VB or Se $4 s$ core-level shift reaffirms that the effect of space charge or surface photovoltage is negligible. Finally, the effect of laser-assisted photoemission is minimized by the choice of $s$ polarization for the pump. All these observations are consistent with the electronic excitation as the origin of the line shape renormalization. Nevertheless, we cannot exclude a small contribution of the aforementioned effects to the observed dynamics.

Based on the Mott transition interpretation and the assumption that screening by excitons is negligible compared to QFCs [2], we can effectively disentangle both of these populations, as presented in the Fig. 3(c) [28]. The 
result indicates that the excitonic population reaches the critical value within the pump pulse envelope, which is then followed by a rapid decay with a lifetime of $\tau_{1}=98(43)$ fs. The remaining exciton population decays at a much lower rate $\left[\tau_{2}=687(289)\right.$ fs]. In contrast, the QFC population continues to rise even after excitation as a result of exciton dissociation, and decays with a lifetime $\tau=748(50)$ fs. Interestingly, this decay seems to correlate with the slow component of exciton decay that is likely due to diffusion into the bulk, as excited-carrier lifetimes are typically observed on the $\sim 100 \mathrm{ps}$ timescale [45].

Previous experimental studies of the excitonic Mott transition in TMDCs have observed either continuous [42] or discontinuous behavior [46], while theory predicts that both of these cases can be realized depending on the interaction strength [3]. Our results shed new light into the ultrafast dynamics of the exciton-QFC transition [see Fig. 3(d)]. The observed degree of ionization $\alpha=n_{\mathrm{QFC}} / n$ stays in the range of $0.2-1$ for the first 1 ps after photoexcitation. For longer time delays, the excited carriers are found exclusively in the QFC state. The observation is indicative of a continuous transition with coexisting phases. It should be underlined that details of the transition will depend on the excitation density. Additional data acquired simultaneously, but with $25 \%$ lower pump fluence indicate that both $\alpha$ and the initial decay of exciton population depend on the total excitation density, corroborating our data interpretation [28].

The possibility to disentangle the dynamics of excitons and QFCs by trXPS is quite surprising due to the small energy difference between these two phases, typically $\sim 50 \mathrm{meV}$, in comparison to the characteristic core-state energy scale of tens of eV. However, it was shown before that small changes in the valence band structure can have a dramatic effect on the shape of core-level spectra [19]. Discrimination of excitons and QFCs is based on the different screening of the core-hole created during the photoemission process (see Fig 4). This can be understood quite intuitively-excitons, being localized charge dipoles, are expected to interact much more weakly with the suddenly created core-hole potential than delocalized QFCs. The most cogent manifestation of this effect is the $\sim 100$ fs delay of the core-level peak-position shift with respect to the peak-width increase [see Fig. 3(a) for the dynamics of closely related parameters], which is connected to the time needed for the critical density of excitons to be reached. Our results support the continuous phase transition scenario of the excitonic Mott transition in TMDC materials. The detailed interpretations of these results rely on the proposed theoretical model which, we believe, is readily applicable to further ultrafast studies of many-body states as well as electronic phase transitions. The advantage of the trXPS approach over optical spectroscopy techniques is element-specific information. This seems to be especially appealing in combination with hard x-ray photoemission due to its larger probing depth, providing access to buried interfaces in realistic semiconducting devices or heterostructures.

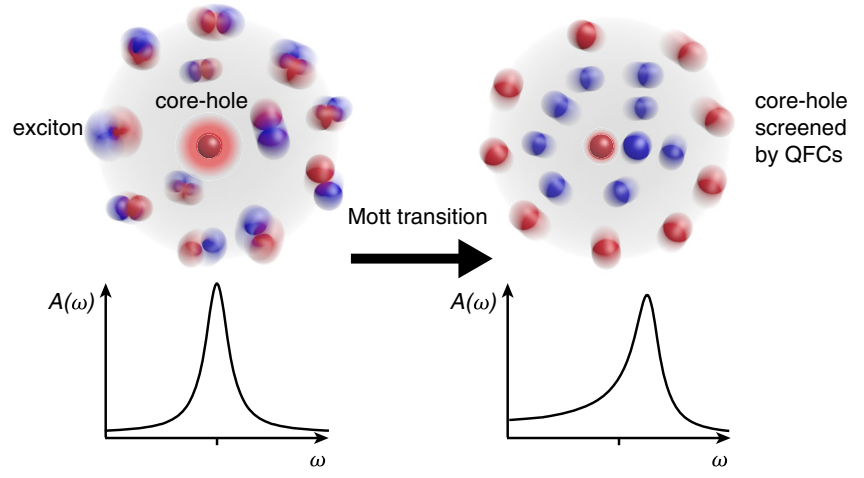

FIG. 4. Illustration of the effect of excitons and QFCs on the core-hole line shape. Charge-neutral electron-hole pairs only weakly screen the core holes and have a negligible effect on its spectral function (left). In contrast, single-particle-like QFCs more effectively screen the localized charge residing in the core hole, resulting in a renormalization of the photoemission line shape (right).

All photoemission data used for the presented analysis are publicly available [47].

We acknowledge support by the scientific and technical staff of FLASH as well as Holger Meyer and Sven Gieschen from the University of Hamburg and valuable discussions with Karsten Horn and Lucia Reining. This work was funded by the European Research Council (ERC) under the European Union's Horizon 2020 research and innovation program (Grant No. ERC-2015-CoG-682843), the German Research Foundation (DFG) within the Emmy Noether program (Grant No. RE 3977/1), through the SFB/ TRR 227 "Ultrafast Spin Dynamics" (projects A09 and B07), the SFB 925 "Light Induced Dynamics and Control of Correlated Quantum Systems" (project B2), TRR 173 "Spin+X: Spin in its collective environment" (project A05), and the Max Planck Society. F. P. acknowledges funding from the excellence cluster "The Hamburg Centre for Ultrafast Imaging-Structure, Dynamics and Control of Matter at the Atomic Scale" of the Deutsche Forschungsgemeinschaft (DFG EXC 1074). A. M. and D.S. acknowledge the funding received from the European Union projects: MaX Materials design at the eXascale H2020-EINFRA-2015-1, Grant No. 676598, and H2020-INFRAEDI-2018-2020/H2020-INFRAEDI2018-1, Grant No. 824143; Nanoscience Foundries and Fine Analysis - Europe (NFFA-Europe) H2020-INFRAIA2014-2015, Grant No. 654360. M. D., R. E. and L. R. acknowledge funding from NFFA-Europe (Project No. 879). G. S., D. S., A. M., and E.P. acknowledge funding from MIUR PRIN Grant No. 20173B72NB. S. B. acknowledges financial support from the Natural Sciences and Engineering Research Council of Canada Banting Postdoctoral Fellowships Program. This work was supported by VILLUM FONDEN via the Centre of Excellence for Dirac Materials (Grant No. 11744). 
*Deceased.

†dendzik@kth.se

*ernstorfer@fhi-berlin.mpg.de

[1] T. Mueller and E. Malic, 2D Mater. Appl. 2, 29 (2018).

[2] A. Steinhoff, M. Florian, M. Rösner, G. Schönhoff, T. O. Wehling, and F. Jahnke, Nat. Commun. 8, 1166 (2017).

[3] D. Guerci, M. Capone, and M. Fabrizio, Phys. Rev. Mater. 3, 054605 (2019).

[4] R. A. Kaindl, M. A. Carnahan, D. Hägele, R. Lövenich, and D. S. Chemla, Nature (London) 423, 734 (2003).

[5] R. Huber, F. Tauser, A. Brodschelm, M. Bichler, G. Abstreiter, and A. Leitenstorfer, Nature (London) 414, 286 (2001).

[6] A. Pietzsch, A. Föhlisch, M. Beye, M. Deppe, F. Hennies, M. Nagasono, E. Suljoti, W. Wurth, C. Gahl, K. Döbrich et al., New J. Phys. 10, 033004 (2008).

[7] G. Stefanucci and R. van Leeuwen, Nonequilibrium Many-Body Theory of Quantum Systems: A Modern Introduction (Cambridge University Press, Cambridge, 2013), ISBN 9781139023979.

[8] G. Ertl and N. Thiele, Appl. Surf. Sci. 3, 99 (1979).

[9] G. Ertl, R. Hierl, H. Knözinger, N. Thiele, and H. Urbach, Appl. Surf. Sci. 5, 49 (1980).

[10] R. Asahi, T. Morikawa, T. Ohwaki, K. Aoki, and Y. Taga, Science 293, 269 (2001).

[11] M. C. Biesinger, B. P. Payne, A. P. Grosvenor, L. W. Lau, A. R. Gerson, and R. S. Smart, Appl. Surf. Sci. 257, 2717 (2011).

[12] Z. Xu, L.-M. Chen, G. Yang, C.-H. Huang, J. Hou, Y. Wu, G. Li, C.-S. Hsu, and Y. Yang, Adv. Funct. Mater. 19, 1227 (2009).

[13] P. H. Citrin, G. K. Wertheim, and Y. Baer, Phys. Rev. B 16, 4256 (1977).

[14] S. Hüfner, Photoelectron Spectroscopy (Springer, Berlin, Heidelberg, 2003), ISBN 9783642075209.

[15] G. D. Mahan, Many-Particle Physics (Springer US, New York, 2000), ISBN 9781475757149.

[16] S. Lizzit, K. Pohl, A. Baraldi, G. Comelli, V. Fritzsche, E. W. Plummer, R. Stumpf, and P. Hofmann, Phys. Rev. Lett. 81, 3271 (1998).

[17] S. Doniach and M. Šunjić, J. Phys. C 3, 285 (1970).

[18] M. Dendzik, M. Michiardi, C. Sanders, M. Bianchi, J. A. Miwa, S. S. Grønborg, J. V. Lauritsen, A. Bruix, B. Hammer, and P. Hofmann, Phys. Rev. B 92, 245442 (2015).

[19] M. Dendzik, A. Bruix, M. Michiardi, A. S. Ngankeu, M. Bianchi, J. A. Miwa, B. Hammer, P. Hofmann, and C. E. Sanders, Phys. Rev. B 96, 235440 (2017).

[20] W. Ackermann, G. Asova, V. Ayvazyan, A. Azima, N. Baboi, J. Bähr, V. Balandin, B. Beutner, A. Brandt, A. Bolzmann et al., Nat. Photonics 1, 336 (2007).

[21] G. Schönhense, K. Medjanik, and H.-J. Elmers, J. Electron Spectrosc. Relat. Phenom. 200, 94 (2015).

[22] S. Hellmann, M. Beye, C. Sohrt, T. Rohwer, F. Sorgenfrei, H. Redlin, M. Kalläne, M. Marczynski-Bühlow, F. Hennies, M. Bauer et al., Phys. Rev. Lett. 105, 187401 (2010).

[23] K. Ishizaka, T. Kiss, T. Yamamoto, Y. Ishida, T. Saitoh, M. Matsunami, R. Eguchi, T. Ohtsuki, A. Kosuge, T. Kanai et al., Phys. Rev. B 83, 081104(R) (2011).
[24] K. R. Siefermann, C. D. Pemmaraju, S. Neppl, A. Shavorskiy, A. A. Cordones, J. Vura-Weis, D. S. Slaughter, F. P. Sturm, F. Weise, H. Bluhm et al., J. Phys. Chem. Lett. 5, 2753 (2014).

[25] R.-Y. Liu, K. Ozawa, N. Terashima, Y. Natsui, B. Feng, S. Ito, W.-C. Chen, C.-M. Cheng, S. Yamamoto, H. Kato et al., Appl. Phys. Lett. 112, 211603 (2018).

[26] R. Bertoni, C. W. Nicholson, L. Waldecker, H. Hübener, C. Monney, U. De Giovannini, M. Puppin, M. Hoesch, E. Springate, R. T. Chapman et al., Phys. Rev. Lett. 117, 277201 (2016).

[27] M. Puppin, Freie Universität Berlin, Ph. D. thesis, 2018, http://dx.doi.org/10.17169/refubium-804.

[28] See Supplemental Material at http://link.aps.org/ supplemental/10.1103/PhysRevLett.125.096401 for additional information on the experimental details, theoretical model derivation, model-independent analysis, excitation density estimation, and data processing, which includes Refs. [29-34].

[29] A. Gerlach, K. Berge, A. Goldmann, I. Campillo, A. Rubio, J. M. Pitarke, and P. M. Echenique, Phys. Rev. B 64, 085423 (2001).

[30] F. Borgatti, J. A. Berger, D. Céolin, J. S. Zhou, J. J. Kas, M. Guzzo, C. F. McConville, F. Offi, G. Panaccione, A. Regoutz et al., Phys. Rev. B 97, 155102 (2018).

[31] E. Perfetto, D. Sangalli, A. Marini, and G. Stefanucci, Phys. Rev. B 94, 245303 (2016).

[32] S. Schulz, I. Grguraš, C. Behrens, H. Bromberger, J. T. Costello, M. K. Czwalinna, M. Felber, M. C. Hoffmann, M. Ilchen, H. Y. Liu et al., Nat. Commun. 6, 5938 (2015).

[33] A. R. Beal, W. Y. Liang, and H. P. Hughes, J. Phys. C 9, 2449 (1976).

[34] M. Dresselhaus, G. Dresselhaus, S. Cronin, and A. G. S. Filho, Solid State Properties (Springer, Berlin, Heidelberg, 2018), ISBN 9783662559222.

[35] D. Kutnyakhov, R. P. Xian, M. Dendzik, M. Heber, F. Pressacco, S. Y. Agustsson, L. Wenthaus, H. Meyer, S. Gieschen, G. Mercurio et al., Rev. Sci. Instrum. 91, 013109 (2020).

[36] P. Nozières, J. Gavoret, and B. Roulet, Phys. Rev. 178, 1084 (1969).

[37] P. Nozières and C. T. De Dominicis, Phys. Rev. 178, 1097 (1969).

[38] L. Hedin, B. Lundqvist, and S. Lundqvist, in Electron Density of States, edited by I. H. Bennet (National Bureau of Standards, Washington, DC, 1971), Special Publication No 323, pp. 233-247.

[39] D. Christiansen, M. Selig, E. Malic, R. Ernstorfer, and A. Knorr, Phys. Rev. B 100, 205401 (2019).

[40] E. Perfetto, D. Sangalli, A. Marini, and G. Stefanucci, Phys. Rev. Mater. 3, 124601 (2019).

[41] P. Steinleitner, P. Merkl, P. Nagler, J. Mornhinweg, C. Schüller, T. Korn, A. Chernikov, and R. Huber, Nano Lett. 17, 1455 (2017).

[42] A. Chernikov, C. Ruppert, H. M. Hill, A. F. Rigosi, and T. F. Heinz, Nat. Photonics 9, 466 (2015).

[43] S. Hellmann, C. Sohrt, M. Beye, T. Rohwer, F. Sorgenfrei, M. Marczynski-Bühlow, M. Kalläne, H. Redlin, F. 
Hennies, M. Bauer et al., New J. Phys. 14, 013062 (2012).

[44] L.-P. Oloff, M. Oura, K. Rossnagel, A. Chainani, M. Matsunami, R. Eguchi, T. Kiss, Y. Nakatani, T. Yamaguchi, J. Miyawaki et al., New J. Phys. 16, 123045 (2014).

[45] S. Tokudomi, J. Azuma, K. Takahashi, and M. Kamada, J. Phys. Soc. Jpn. 76, 104710 (2007).
[46] A. W. Bataller, R. A. Younts, A. Rustagi, Y. Yu, H. Ardekani, A. Kemper, L. Cao, and K. Gundogdu, Nano Lett. 19, 1104 (2019).

[47] M. Dendzik, R. P. Xian, E. Perfetto, D. Sangalli, D. Kutnyakhov, S. Dong, S. Beaulieu, T. Pincelli, F. Pressacco, D. Curcio et al., Time-resolved core-level photoemission data of tungsten diselenide (2020) https://doi.org/10.5281/zenodo. 3945433 . 